\title{
Model-based estimates of the finite population mean for two-stage cluster samples with unit non-response
}

\author{
Ying Yuan \\ University of Texas M. D. Anderson Cancer Center, Houston, USA \\ and Roderick J. A. Little \\ University of Michigan, Ann Arbor, USA
}

[Received August 2005. Final revision August 2006]

\begin{abstract}
Summary. We propose new model-based methods for unit non-response in two-stage survey samples. A commonly used design-based adjustment weights respondents by the inverse of the estimated response rate in each cluster (method WT). This approach is consistent if the response probabilities are constant within clusters but is potentially inefficient when the estimated cluster response rates are very variable. Clusters can be collapsed to increase precision, but this may introduce bias. We consider here the model-based approach to survey inference that treats the clusters as random effects. We note that, from a model-based perspective, a missing data mechanism that assumes that the response rate varies across clusters is non-ignorable, and we propose the term cluster-specific non-ignorable (CSNI) non-response to describe this mechanism. We show that the standard random-effects model estimator RE of the population mean is biased under CSNI non-response, and we propose two modifications of RE to correct this bias. One approach includes the observed response rate as a cluster level covariate (method RERR), and the other is based on a probit model for response (method NI1). The RERR approach is simpler than NI1 but approximate, in that uncertainty in estimating the response rates is not taken into account. In addition, a simple method that corrects the bias of RE by reweighting (method RWRE) is also discussed. We show by simulations that estimators from RERR and NI1 can correct the bias of RE under CSNI non-response and have comparable or lower root-mean-squared error than WT in a variety of simulation settings, and RWRE has similar performance to WT. We also consider another non-ignorable response model estimate of the population mean (NI2) that removes the bias of WT, RWRE, RERR and NI1 under an outcome-specific non-ignorable response mechanism where non-response depends directly on the individual level survey outcomes. However, that estimate is not robust to model misspecification. The various methods are compared on a data set from the Detroit Dental Health Project.
\end{abstract}

Keywords: Cluster sampling; Non-ignorable non-response; Random-effects model; Unit non-response

\section{Introduction}

Statistical adjustments for non-response are increasingly important, given declining response rates in many surveys (De Heer, 1999; Groves and Couper, 1998). This paper concerns unit non-response adjustments in the context of two-stage cluster sampling. Consider a population of size $M$ consisting of $N$ clusters with $M_{i}$ elements in the $i$ th cluster. Let $Y_{i j}$ denote the value

Address for correspondence: Roderick J. A. Little, Department of Biostatistics, University of Michigan, 1420 Washington Heights, Ann Arbor, MI 48109, USA.

E-mail: rlittle@umich.edu 
of a survey outcome $Y$ for unit $j$ in cluster $i$, for $i=1, \ldots, N, j=1, \ldots, M_{i}$, and let

$$
T=\sum_{i=1}^{N} \sum_{j=1}^{M_{i}} Y_{i j}
$$

and $\bar{Y}=T / M$ denote the population total and mean respectively. At the first stage, a sample of $n$ of the $N$ clusters (primary sampling units (PSUs)) is selected. At the second stage, $m_{i}$ of the $M_{i}$ units (secondary sampling units (SSUs)) are selected in the $i$ th sampled cluster, but only $r_{i}$ of the $m_{i}$ sampled units respond. We consider estimates of the finite population mean $\bar{Y}$ or total $T$. To focus on non-response adjustment, we assume that survey sampling is non-informative in the sense that the selection probability of a unit does not depend on its value of $Y$. A commonly used design-based approach is to discard the non-respondents and to weight the respondents in the $i$ th cluster by the design weight multiplied by the inverse of its response rate $r_{i} / m_{i}$ in the cluster. This weighting approach is simple, and design consistent if response probabilities are constant within clusters. A weakness of this weighting estimate is that it may have large variance if the weights $m_{i} / r_{i}$ are highly variable; it is not even defined if $r_{i}=0$ for one or more clusters $i$. In addition, it is well known that the weighting adjustment yields a biased estimate of $\bar{Y}$ if the response probability of the $j$ th subject in the $i$ th cluster depends on the survey outcome $y_{i j}$.

An alternative approach is to apply a random-effects model to the respondent data, such as the model for two-stage samples that was proposed by Scott and Smith (1969). This approach addresses the potential inefficiency of the weighting estimate by borrowing strength across clusters. Unfortunately, we show below that this approach requires that the missing data are missing completely at random, and in particular it does not in general yield a consistent estimate of the population mean if the non-response rates vary across the clusters. This fact motivates extensions of the Scott and Smith (1969) model that allow the outcome mean to depend on the non-response rate in each cluster. A simulation study is described that compares the estimates and inferences from these models with existing approaches.

Rubin (1976) and Little and Rubin (2002) classified non-response mechanisms into three types: missingness completely at random (MCAR), when the probability of the non-response does not depend on the clusters or survey variables; missingness at random (MAR), when the probability of response depends only on the observed values; non-ignorable non-response, when the probability of the non-response depends on the unobserved values. In the context of two-stage samples, three non-response mechanisms that are of particular interest are

(a) MCAR non-response,

(b) cluster-specific non-response, where the probability of response depends on cluster characteristics but not on survey outcomes within clusters, and

(c) outcome-specific non-response that depends directly on the value of missing survey outcomes.

It is clear that outcome-specific non-response is non-ignorable. More surprisingly, we point out that cluster-specific non-response is also non-ignorable in the context of the random-effects models that are considered here.

Section 2 describes the design-based weighting method, the estimator of the population mean that results from a standard application of the Scott and Smith model and our proposed extensions. Section 3 describes a simulation study to compare the estimators. Section 4 compares various methods on data from the Detroit Dental Health Project. Section 5 discusses our findings and gives directions for future work. 


\section{Weighting and model-based methods}

\subsection{Weighting methods}

A standard estimate of $\bar{Y}$ for two-stage samples with complete response is the Horvitz-Thompson (Horvitz and Thompson, 1952) estimator, which weights cases by the inverse of their probability of selection. With non-response, a naïve approach is to apply this estimator to the respondents, yielding

$$
\bar{y}_{\mathrm{HT}}=\sum_{i=1}^{n} \sum_{j=1}^{r_{i}} \pi_{i j}^{-1} y_{i j} / \sum_{i=1}^{n} \sum_{j=1}^{r_{i}} \pi_{i j}^{-1},
$$

where $\pi_{i j}$ is the selection probability of unit $j$ in cluster $i$, which is determined by the survey design. This approach generally requires the missing data mechanism to be MCAR to be consistent. A popular modification for unit non-response multiplies the sampling weight for respondents by a response weight given by the inverse of the estimated response rate within a weighting class, which is often chosen as the cluster for two-stage cluster samples. Respondent $j$ in cluster $i$ then receives a weight

$$
w_{i j}=\left(\pi_{i j} \hat{\phi}_{i}\right)^{-1}
$$

where $\hat{\phi}_{i}=r_{i} / m_{i}$ is the observed response rate in cluster $i$. We assume here that the sampling weight is constant within clusters, i.e. $\pi_{i j}=\pi_{i}$ for all $j$; for a discussion of the case where the sampling weight varies within clusters, see Little and Vartivarian (2002). The weighting estimate WT of $\bar{Y}$ is then

$$
\bar{y}_{\mathrm{WT}}=\sum_{i=1}^{n} y_{i .}^{*} / \sum_{i=1}^{n} \pi_{i .}^{*}
$$

where $y_{i .}^{*}=\left(\pi_{i} \hat{\phi}_{i}\right)^{-1} r_{i} \bar{y}_{i}, \pi_{i .}^{*}=r_{i}\left(\pi_{i} \hat{\phi}_{i}\right)^{-1}$ and $\bar{y}_{i}$ is the respondent mean in cluster $i$.

The variance of $\bar{y}_{\mathrm{WT}}$ is approximated by

$$
V\left(\bar{y}_{\mathrm{WT}}\right)=\frac{1}{\left(\sum_{i=1}^{n} \pi_{i .}^{*}\right)^{2}}\left\{V\left(\sum_{i=1}^{n} y_{i .}^{*}\right)+\bar{y}_{\mathrm{WT}}^{2} V\left(\sum_{i=1}^{n} \pi_{i .}^{*}\right)-2 \bar{y}_{\mathrm{WT}} \operatorname{cov}\left(\sum_{i=1}^{n} y_{i .}^{*}, \sum_{i=1}^{n} \pi_{i .}^{*}\right)\right\} .
$$

A consistent estimate of $V\left(\bar{y}_{\mathrm{WT}}\right)$ is obtained by replacing the variances and covariances in equation (4) with sample estimates.

The methods that are described here apply to any two-stage sampling scheme, but in our simulations we confine attention to the common design that selects PSUs with probability proportional to PSU size, and SSUs with probability inversely proportional to PSU size (the PPS-inverse PPS design), yielding a design with constant inclusion probabilities $\pi_{i j}=\pi$ for all $i$ and $j$ (Kish, 1965). The estimate (1) for this design simplifies to the unweighted mean UW,

$$
\bar{y}_{\mathrm{UW}}=\sum_{i=1}^{n} r_{i} \bar{y}_{i} / \sum_{i=1}^{n} r_{i},
$$

and the weighting estimate (3) is

$$
\bar{y}_{\mathrm{WT}}=n^{-1} \sum_{i=1}^{n} \bar{y}_{i}
$$

WT is simple and corrects the bias of equation (1) when the response probabilities are constant within clusters, but it has some drawbacks. First, WT tends to have a large variance if 
the sample response rates are very variable. In the extreme, WT is undefined when one or more clusters do not have any respondent. Ways around this difficulty are to compute WT by using only clusters with respondents, or to combine clusters with no respondents with other clusters, but both approaches may lead to bias. A second limitation of WT is that it is biased if the response probability of the $i j$ th population member depends on the value of $y_{i j}$. In the next section we describe model-based methods for addressing these limitations.

\subsection{Random-effects models}

An alternative to WT is to discard non-respondents and to base estimates on predictions from a random-effects model fitted to respondents. A natural model for two-stage samples is that first proposed by Scott and Smith (1969) (method RE), namely

$$
\begin{aligned}
{\left[y_{i j} \mid \mu_{i}, \sigma^{2}\right] } & =N\left(\mu_{i}, \sigma^{2}\right), \\
{\left[\mu_{i} \mid \mu, \tau^{2}\right] } & =N\left(\mu, \tau^{2}\right),
\end{aligned}
$$

where $N(\cdot)$ denotes a normal distribution. RE resolves the potential inefficiency of WT, and the lack of definition of WT when there are clusters with no respondents, by borrowing strength across clusters. Let $\hat{\sigma}^{2}$ and $\hat{\tau}^{2}$ denote estimates of the fixed parameters, computed for example by restricted maximum likelihood. For the PPS-inverse PPS design that we consider, larger PSUs are overrepresented in the sample, and valid application of this model requires the assumption that the cluster means are unrelated to size (Sverchkov and Pfeffermann, 2004). To focus on the non-response issue, we make that assumption here and comment on the case where this does not hold in the concluding section. For purposes of prediction under model (5), units of the population fall into three groups. The first group consists of the respondent values, say $\mathbf{y}_{\mathrm{obs}}$, which are known and do not require estimation. The second group consists of the units in clusters that are not sampled; these are predicted by the estimate of $\mu$, which is a weighted mean:

$$
\begin{aligned}
\bar{y}_{\mathrm{W}} & =\sum_{i=1}^{n} \kappa_{i} \bar{y}_{i} / \sum_{i=1}^{n} \kappa_{i}, \\
\kappa_{i} & =\frac{\hat{\tau}^{2}}{\hat{\tau}^{2}+\hat{\sigma}^{2} / r_{i}} .
\end{aligned}
$$

The third group consists of the non-respondents and non-sampled units in the sampled clusters; these are estimated by

$$
E\left(\mu_{i} \mid \mathbf{y}_{\mathrm{obs}}\right)=\kappa_{i} \bar{y}_{i}+\left(1-\kappa_{i}\right) \bar{y}_{\mathrm{W}} .
$$

Combining these predictions, $\bar{Y}$ is estimated by

$$
\begin{aligned}
\bar{y}_{\mathrm{RE}} & =E\left(\bar{Y} \mid \mathbf{y}_{\mathrm{obs}}\right) \\
& =\frac{1}{M}\left[\sum_{i=1}^{n} \sum_{j=1}^{r_{i}} y_{i j}+\sum_{i=1}^{n}\left(M_{i}-r_{i}\right)\left\{\kappa_{i} \bar{y}_{i}+\left(1-\kappa_{i}\right) \bar{y}_{\mathrm{W}}\right\}+\sum_{i=n+1}^{N} M_{i} \bar{y}_{\mathrm{W}}\right] .
\end{aligned}
$$

The estimated variance of $\bar{y}_{\mathrm{RE}}$ is given by Scott and Smith (1969).

If the non-response mechanism is MCAR, $\bar{y}_{\mathrm{RE}}$ has similar properties to the RE estimator without non-response, i.e. $\bar{y}_{\mathrm{RE}}$ is consistent and is the best linear unbiased predictor of $\bar{Y}$ if the model is correctly specified (Scott and Smith, 1969). If the non-response probability of $y_{i j}$ depends on cluster characteristics, and in particular the cluster means $\mu_{i}$, we might think that the data are missing at random since cluster membership is observed for non-respondents. However, the missing data mechanism is in fact not MAR, since the cluster means are unobserved 
random effects; we propose the term cluster-specific non-ignorable (CSNI) non-response to describe this mechanism, to distinguish it from other forms of non-ignorable non-response. A consequence of the non-ignorability is that RE leads to biased estimates in this case (Little and Rubin (2002), example 6.24). The bias comes from two sources. First, $\bar{y}_{\mathrm{W}}$ is a biased estimate of $\mu$. To see this, we rewrite $\bar{y}_{\mathrm{W}}$ as

$$
\bar{y}_{\mathrm{W}}=\sum_{i=1}^{n} \frac{\hat{\tau}^{2}}{\hat{\tau}^{2}+\left(\hat{\sigma}^{2} / m_{i}\right)\left(1 / \hat{\phi}_{i}\right)} \bar{y}_{i} / \sum_{i=1}^{n} \frac{\hat{\tau}^{2}}{\hat{\tau}^{2}+\left(\hat{\sigma}^{2} / m_{i}\right)\left(1 / \hat{\phi}_{i}\right)} .
$$

Since the non-response probability of $y_{i j}$ depends only on the underlying cluster means $\mu_{i}$, respondents in cluster $i$ are a random sample of elements in this cluster. Thus, $\bar{y}_{i}$ is a consistent estimate of $\mu_{i}$, and the correct weight associated with $\bar{y}_{i}$ is

$$
\kappa_{i}=\frac{\hat{\tau}^{2}}{\hat{\tau}^{2}+\hat{\sigma}^{2} / m_{i}} .
$$

However, the weight $\kappa_{i}$ here is distorted by the different cluster response rate $\hat{\phi}_{i}$, so $\bar{y}_{\mathrm{W}}$ is biased. The larger is the variation of $\hat{\phi}_{i}$, the larger the bias. Second, the predictor of non-respondents and non-sampled elements, $\kappa_{i} \bar{y}_{i}+\left(1-\kappa_{i}\right) \bar{y}_{\mathrm{W}}$, adjusts the cluster sample mean towards this biased estimate of $\mu$. The extent of the adjustment is determined by $\hat{\sigma}^{2} / \hat{\tau}^{2}$ and $r_{i}$. If $r_{i}$ is very large, $\kappa_{i} \approx 1$, and there is very little adjustment. If $r_{i}$ is small, large $\hat{\sigma}^{2} / \hat{\tau}^{2}$ causes more adjustment, and hence more bias. These facts motivate a simple approach to correct the bias by modifying $\kappa_{i}$ to reflect the correct weight. Specifically, we change $\kappa_{i}$ in equation (6) to that in equation (7). This reweighted random-effects model based approach (method RWRE) yields a consistent estimate of $\bar{Y}$ when non-response depends on cluster means, given that each sampled cluster has at least one respondent. However, if some sampled clusters do not have any respondent, RWRE yields biased estimates, since it ignores these clusters.

The bias of RE when data are not missing completely at random motivates extensions of the RE model for CSNI mechanisms. An informal approach is to modify the RE model to allow the cluster mean to depend on the estimated cluster response rate. Assuming for simplicity a linear relationship yields the approximate model

$$
\begin{gathered}
{\left[y_{i j} \mid \alpha_{i}, \hat{\phi}_{i}, \sigma^{2}\right]=N\left(\alpha_{i}+\beta \hat{\phi}_{i}, \sigma^{2}\right),} \\
{\left[\alpha_{i} \mid \alpha, \tau^{2}\right]=N\left(\alpha, \tau^{2}\right),}
\end{gathered}
$$

which is easily fitted by including the estimated response rate in each cluster as a covariate in the RE model. We label estimates for this model RERR, for the random-effects model with response rate as covariate. This approach is approximate in that the sampling error in estimating the response rate is not taken into account. A more rigorous extension of RE is to model the missing data mechanism directly by the following non-ignorable probit model (NI1):

$$
\begin{aligned}
& {\left[y_{i j} \mid \alpha_{i}, \chi_{i}, \beta, \sigma^{2}\right]=N\left(\alpha_{i}+\beta \chi_{i}, \sigma^{2}\right),} \\
& {\left[z_{i j} \mid y_{i j}, \alpha_{i}, \chi_{i}\right]=N\left(\chi_{i}, 1\right),} \\
& \begin{array}{c}
r_{i j}=\left\{\begin{array}{lc}
1 & \text { if } z_{i j}>0, \\
0 & \text { if } z_{i j}<0,
\end{array}\right\} \\
{\left[\alpha_{i} \mid \chi_{i}, \alpha, \tau^{2}\right]=N\left(\alpha, \tau^{2}\right),} \\
{\left[\chi_{i} \mid \chi, \omega^{2}\right]=N\left(\chi, \omega^{2}\right),}
\end{array}
\end{aligned}
$$


where $r_{i j}$ is the response indicator. $r_{i j}=1$ for respondents and $r_{i j}=0$ for non-respondents. The normal latent variable $z_{i j}$ determines the response status of the subject $j$ in the cluster $i$. If the value of $z_{i j}$ is greater than the threshold 0 , the subject responds; otherwise, the subject does not respond. The variance of $z_{i j}$ is set to 1 since the scale of this latent variable is arbitrary and not identifiable. Integrating over the latent $\left\{z_{i j}\right\}$ is easily seen to yield a probit model for the missing data process. The latent variable interpretation of the probit model has been discussed by Albert and Chib (1993).

These models assume a CSNI mechanism where $r_{i j}$ and $y_{i j}$ are unrelated within clusters. An outcome-specific non-ignorable (OSNI) response mechanism assumes that missingness of $y_{i j}$ depends directly on the value of $y_{i j}$. Such a mechanism is modelled by changing NI1 so that $y_{i j}$ has a linear regression on $z_{i j}$ rather than its mean $\chi_{i}$, i.e.

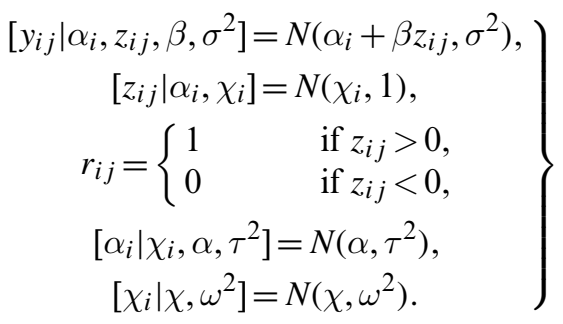

We label this model NI2. This model can be viewed as an extension of the Tobit model (Amemiya, 1984), which is commonly used in econometrics, with random effects to account for the correlation of values of survey outcomes of participants in the same cluster. As in many non-ignorable response models, such as Heckman-type selection models (Heckman, 1976), the estimation of $\beta$ here is heavily driven by the normality assumption of the latent variable and linear relationship between $y_{i j}$ and $z_{i j}$. Since these assumptions are untestable on the basis of the observed data, subject-matter knowledge or external information may be required to justify the assumptions.

Models (8) and (9) are special cases of the more general model

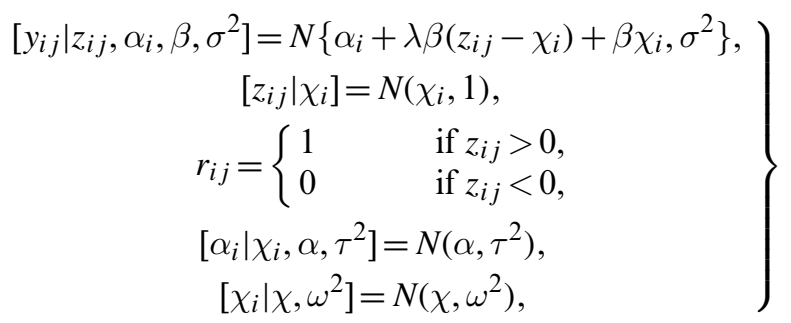

which yields model (8) when $\lambda=0$ and model (9) when $\lambda=1$. It is tempting to try to fit model (10) to the data, but in practice the parameter $\lambda$ is at best weakly identified. This model might form the basis of a sensitivity analysis with a range of fixed values of $\lambda$, and it is used to generate data for the simulation study in Section 3.

\subsection{Fitting the random-effects models}

We could fit the models that are described in Section 2.2 by maximum likelihood. However, a convenient alternative approach that also provides estimates of precision is to add non-informative priors for the fixed parameters, and to simulate draws from the posterior distribution of the parameters. For reviews of the Bayesian approach to sample surveys, see for example Little (2003, 2004). The Bayesian estimate that is discussed here is asymptotically equivalent to the 
maximum likelihood estimate (Gelman et al., 2004). Estimates for the RE and RERR models are obtained by the Gibbs sampler (Gelfand et al., 1990). The Gibbs sampler for RE is easily modified for RWRE by applying the modified weights. For example, for RE, $\mu_{i}$ is drawn from

$$
\left[\mu_{i} \mid y_{i j}, \mu, \sigma^{2}, \tau^{2}\right]=N\left(\frac{\tau^{2}}{\tau^{2}+\sigma^{2} / r_{i}} \bar{y}_{i}+\frac{\sigma^{2} / r_{i}}{\tau^{2}+\sigma^{2} / r_{i}} \mu, \frac{\tau^{2} \sigma^{2}}{r_{i} \tau^{2}+\sigma^{2}}\right) .
$$

In contrast, for RWRE, $\mu_{i}$ is drawn from

$$
\left[\mu_{i} \mid y_{i j}, \mu, \sigma^{2}, \tau^{2}\right]=N\left(\frac{\tau^{2}}{\tau^{2}+\sigma^{2} / m_{i}} \bar{y}_{i}+\frac{\sigma^{2} / m_{i}}{\tau^{2}+\sigma^{2} / m_{i}} \mu, \frac{\tau^{2} \sigma^{2}}{m_{i} \tau^{2}+\sigma^{2}}\right) .
$$

We use non-informative priors for $\mu$ in RE, or $\alpha$ and $\beta$ in RERR, and diffuse inverse gamma priors for $\sigma^{2}$ and $\tau^{2}$. In particular for RERR

$$
\left[\alpha, \beta, \sigma^{2}, \tau^{2}\right] \propto\left(\sigma^{2}\right)^{-\left(a_{1}+1\right)} \exp \left(-b_{1} / \sigma^{2}\right)\left(\tau^{2}\right)^{-\left(a_{2}+1\right)} \exp \left(-b_{2} / \tau^{2}\right)
$$

with $a_{1}=b_{1}=a_{2}=b_{2}=0.1$, a value that is sufficiently small that the information in the data strongly dominates the information in the prior distribution. The variance estimator based on the RERR model might fail since RERR is an approximate model. We hence consider a bootstrap variance estimator for RERR, which is less reliant on the model and more consistent with design-based perspectives. For the models NI1 and NI2 we assume priors for the fixed parameters of the form

$$
\begin{gathered}
{\left[\beta, \sigma^{2}\right] \propto\left(\sigma^{2}\right)^{-\left(a_{1}+1\right)} \exp \left(-b_{1} / \sigma^{2}\right),} \\
{\left[\alpha, \tau^{2}\right] \propto\left(\tau^{2}\right)^{-\left(a_{2}+1\right)} \exp \left(-b_{2} / \tau^{2}\right),} \\
{\left[\chi, \omega^{2}\right] \propto\left(\omega^{2}\right)^{-\left(a_{3}+1\right)} \exp \left(-b_{3} / \omega^{2}\right),}
\end{gathered}
$$

with $a_{1}=b_{1}=a_{2}=b_{2}=a_{3}=b_{3}=0.1$, again so that the information in the data strongly dominates the information in the prior distribution. Computation via the Gibbs sampler is again straightforward, provided that the latent values $\left\{z_{i j}\right\}$ are included as missing data. Details of fitting NI2 are given in Appendix A. We monitored convergence of the Gibbs chains by graphical inspection, and by the methods of Gelman and Rubin (1992) and Johnson (1996).

\section{Simulation study}

\subsection{Description of study}

Eight populations of $M=\Sigma_{i=1}^{N} M_{i}=398886$ values of a variable $Y$ were constructed in 800 clusters. Clusters sizes $\left\{M_{i}\right\}$ were randomly generated from a uniform distribution with a minimum size of 50 and a maximum size of 1000 . The populations were then constructed by using model (10). The parameter $\beta$ determines the degree of non-ignorability of the missing data mechanism. If $\beta=0$, the missing data mechanism is MCAR, and larger values of $\beta$ correspond to stronger degrees of non-ignorability. We simulated populations with two values of $\beta, \beta=0$ and $\beta=10$. The parameter $\lambda$ determines the extent to which the missing data mechanism depends on the cluster mean $\chi_{i}$ and the value of $y_{i j}$ itself. If $\lambda=1$, the missingness of $y_{i j}$ depends entirely on $y_{i j}$, and we have OSNI non-response; if $\lambda=0$, the missingness of $y_{i j}$ only depends on $\chi_{i}$, and we have CSNI non-response. If $0<\lambda<1$, the missingness mechanism is a mixture of CSNI and OSNI non-response. We simulated data with three values of $\lambda: \lambda=0,0.5,1$. The correlation of the outcomes within a cluster is an important characteristic that determines the shrinkage of the random-effects models. We set $\left(\tau^{2}+\beta^{2} \omega^{2}\right) / \sigma^{2}$ equal to $1 / 5$ and 5 to generate a low intracluster correlation of 0.17 and a high intracluster correlation of 0.84 for the outcome values. Since 
Table 1. Empirical bias, RMSE, change in RMSE relative to BD, average estimate of standard error ESTSE and non-coverage rate of $95 \%$ confidence intervals (nominally, target $=25$ ) for seven methods under the $(80,10)$ design $\dagger$

\begin{tabular}{|c|c|c|c|c|c|c|c|c|}
\hline \multicolumn{3}{|c|}{$\begin{array}{l}\text { Simulation } \\
\text { parameters }\end{array}$} & \multirow[t]{3}{*}{ Method } & \multicolumn{5}{|c|}{ Summary outcomes } \\
\hline & & & & \multirow{2}{*}{ Bias $(\times 100)$} & \multirow{2}{*}{$R M S E(\times 100)$} & \multirow{2}{*}{ RRMSE (\%) } & \multirow{2}{*}{$\operatorname{ESTSE}(\times 100)$} & \multirow{2}{*}{$\begin{array}{c}\text { Non-coverage } \\
\text { rate }\end{array}$} \\
\hline$\beta$ & $\lambda$ & CORR & & & & & & \\
\hline \multirow[t]{8}{*}{0} & 0 & $H$ & BD & -1 & 58 & 0 & 59 & 23 \\
\hline & & & UW & 3 & 67 & 14 & 67 & 29 \\
\hline & & & WT & 1 & 64 & 10 & 64 & 30 \\
\hline & & & $\mathrm{RE}$ & -3 & 64 & 9 & 61 & 32 \\
\hline & & & RWRE & 2 & 64 & 10 & 62 & 29 \\
\hline & & & RERR & 2 & 66 & 13 & $69(65)$ & $24(30)$ \\
\hline & & & NI1 & 3 & 66 & 13 & 64 & 28 \\
\hline & & & NI2 & 2 & 120 & 106 & 120 & 27 \\
\hline \multirow[t]{8}{*}{0} & 0 & $L$ & BD & 1 & 102 & 0 & 105 & 22 \\
\hline & & & UW & -5 & 127 & 25 & 129 & 23 \\
\hline & & & WT & 8 & 140 & 37 & 148 & 18 \\
\hline & & & $\mathrm{RE}$ & -6 & 128 & 26 & 121 & 28 \\
\hline & & & RWRE & 8 & 142 & 39 & 145 & 18 \\
\hline & & & RERR & 5 & 147 & 44 & $142(152)$ & $35(21)$ \\
\hline & & & NI1 & 9 & 141 & 38 & 151 & 20 \\
\hline & & & NI2 & 48 & 328 & 221 & 314 & 31 \\
\hline \multirow[t]{8}{*}{10} & 0 & $H$ & BD & 3 & 122 & 0 & 123 & 27 \\
\hline & & & UW & 415 & 431 & 253 & 119 & 467 \\
\hline & & & WT & 81 & 145 & 19 & 122 & 49 \\
\hline & & & RE & 110 & 161 & 32 & 114 & 88 \\
\hline & & & RWRE & 83 & 146 & 20 & 116 & 58 \\
\hline & & & RERR & -1 & 124 & 2 & $90(123)$ & $68(29)$ \\
\hline & & & NI1 & -5 & 126 & 3 & 127 & 29 \\
\hline & & & NI2 & -180 & 227 & 86 & 123 & 154 \\
\hline \multirow[t]{8}{*}{10} & 0 & $L$ & BD & 7 & 146 & 0 & 154 & 21 \\
\hline & & & UW & 455 & 483 & 230 & 165 & 396 \\
\hline & & & WT & 92 & 203 & 38 & 185 & 43 \\
\hline & & & $\mathrm{RE}$ & 326 & 365 & 149 & 164 & 257 \\
\hline & & & RWRE & 94 & 207 & 41 & 182 & 47 \\
\hline & & & RERR & -5 & 183 & 25 & $161(186)$ & $44(29)$ \\
\hline & & & NI1 & 15 & 183 & 25 & 187 & 21 \\
\hline & & & NI2 & -851 & 914 & 525 & 332 & 364 \\
\hline \multirow[t]{8}{*}{10} & 0.5 & $H$ & BD & -3 & 123 & 0 & 128 & 23 \\
\hline & & & UW & 680 & 688 & 458 & 108 & 500 \\
\hline & & & WT & 411 & 423 & 243 & 104 & 491 \\
\hline & & & $\mathrm{RE}$ & 454 & 464 & 277 & 99 & 500 \\
\hline & & & RWRE & 413 & 425 & 245 & 100 & 493 \\
\hline & & & RERR & 343 & 358 & 190 & 86 (104) & 479 (455) \\
\hline & & & NI1 & 339 & 355 & 188 & 106 & 444 \\
\hline & & & NI2 & -23 & 138 & 12 & 125 & 40 \\
\hline \multirow[t]{8}{*}{10} & 0.5 & $L$ & BD & 2 & 159 & 0 & 155 & 26 \\
\hline & & & UW & 688 & 706 & 344 & 155 & 496 \\
\hline & & & WT & 427 & 457 & 188 & 171 & 352 \\
\hline & & & $\mathrm{RE}$ & 613 & 632 & 298 & 151 & 489 \\
\hline & & & RWRE & 431 & 462 & 191 & 169 & 359 \\
\hline & & & RERR & 340 & 378 & 138 & $160(173)$ & $280(251)$ \\
\hline & & & NI1 & 353 & 391 & 146 & 176 & 268 \\
\hline & & & NI2 & -383 & 512 & 222 & 333 & $\begin{array}{c}98 \\
\text { (continued) }\end{array}$ \\
\hline
\end{tabular}


Table 1 (continued)

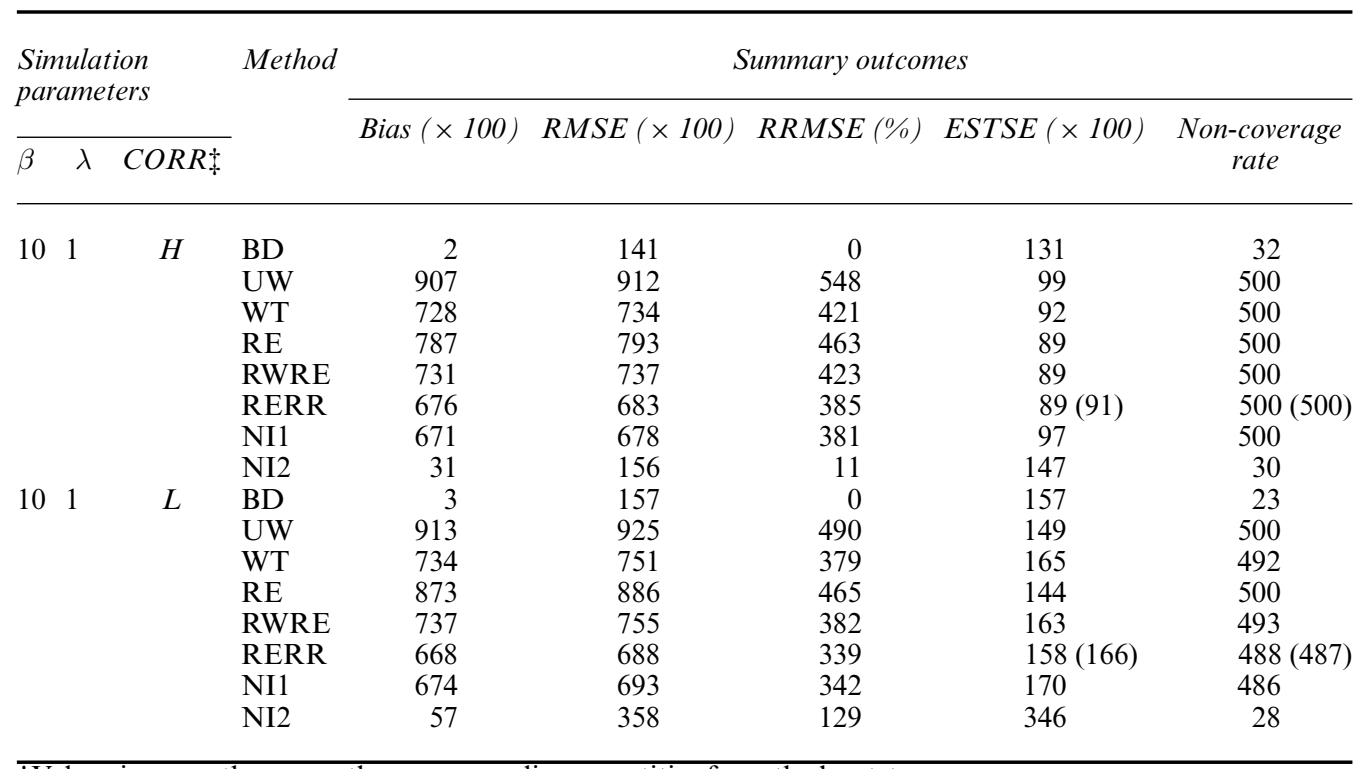

TValues in parentheses are the corresponding quantities from the bootstrap.

$\ddagger H$, high; $L$, low.

the scale of latent variable $z_{i j}$ is unidentifiable and arbitrarily set as 1 , to obtain differential response rates across clusters, we set $\chi=1.4$ and $\omega^{2}=1$. Note that a large value of $\omega^{2}$ leads to many clusters that are either all respondents or no respondents, which may not be of interest in practice. The parameter $\tau^{2}$ is the variance of the random intercept $\alpha_{i}$. We simply set it arbitrarily at 4 since what is of importance is to control the within-cluster correlation as above. The other parameters in model (10) were chosen so that the superpopulation mean is 40 and the overall response rate is $60 \%$.

Two sampling designs were applied to these populations. In the first, which is denoted the $(80,10)$ design, a first-stage sample of $n=80$ PSUs (or clusters) was chosen by probability proportional to size sampling, and a second-stage sample of $m=10$ SSUs (or elements) was selected from each sampled PSU, yielding a total sample size of 800 . In the second design, which is denoted $(20,40), n=20$ PSUs and $m=40$ SSUs were selected in a similar manner. Each sampling scheme was repeated 500 times for each population and the estimate of the finite population mean from each method (UW, WT, RE, RWRE, RERR, NI1 and NI2) was computed. The unweighted mean before deletion of the missing values (method BD) was also computed as a bench-mark, although of course this method would not be available in practice.

\subsection{Results}

Table 1 shows various summary statistics under each model over the 500 samples for the (80, 10) design, including empirical bias, which is the deviation of the average estimate over the 500 samples from the true finite population mean, the root-mean-squared error RMSE, which is the square root of the average squared deviation of the estimate from the true population mean over the 500 samples, the increase in the root-mean-squared error relative to the BD estimator (RRMSE), which is defined as 
Table 2. Empirical bias, RMSE, change in RMSE relative to BD, average estimate of standard error ESTSE and non-coverage rate of $95 \%$ confidence intervals (nominally, target $=25$ ) for seven methods under the $(20,40)$ design†

\begin{tabular}{|c|c|c|c|c|c|c|c|c|}
\hline \multicolumn{3}{|c|}{$\begin{array}{l}\text { Simulation } \\
\text { parameters }\end{array}$} & \multirow[t]{3}{*}{ Method } & \multicolumn{5}{|c|}{ Summary outcomes } \\
\hline & & & & \multirow{2}{*}{$\operatorname{Bias}(\times 100)$} & \multirow{2}{*}{$R M S E(\times 100)$} & \multirow{2}{*}{ RRMSE $(\%)$} & \multirow{2}{*}{$\operatorname{ESTSE}(\times 100)$} & \multirow{2}{*}{$\begin{array}{c}\text { Non-coverage } \\
\text { rate }\end{array}$} \\
\hline$\beta$ & $\lambda$ & CORR & & & & & & \\
\hline \multirow[t]{8}{*}{0} & 0 & $H$ & BD & 11 & 111 & 0 & 114 & 32 \\
\hline & & & UW & 3 & 122 & 10 & 124 & 31 \\
\hline & & & WT & 13 & 116 & 5 & 117 & 32 \\
\hline & & & RE & 11 & 115 & 4 & 124 & 30 \\
\hline & & & RWRE & 13 & 116 & 5 & 125 & 26 \\
\hline & & & RERR & 12 & 116 & 5 & $126(121)$ & $27(30)$ \\
\hline & & & NI1 & 12 & 116 & 5 & 134 & 20 \\
\hline & & & NI2 & 16 & 149 & 34 & 179 & 18 \\
\hline \multirow[t]{8}{*}{0} & 0 & $L$ & BD & 1 & 146 & 0 & 140 & 31 \\
\hline & & & UW & -17 & 171 & 17 & 165 & 30 \\
\hline & & & WT & -1 & 182 & 25 & 181 & 29 \\
\hline & & & RE & -13 & 167 & 14 & 170 & 27 \\
\hline & & & RWRE & 1 & 185 & 27 & 192 & 25 \\
\hline & & & RERR & 0 & 174 & 19 & $175(174)$ & $32(32)$ \\
\hline & & & NI1 & -5 & 171 & 18 & 176 & 29 \\
\hline & & & NI2 & 18 & 345 & 137 & 358 & 22 \\
\hline \multirow[t]{8}{*}{10} & 0 & $H$ & BD & 4 & 235 & 0 & 233 & 23 \\
\hline & & & UW & 443 & 500 & 112 & 233 & 243 \\
\hline & & & WT & 31 & 248 & 5 & 246 & 31 \\
\hline & & & RE & 42 & 246 & 4 & 260 & 27 \\
\hline & & & RWRE & 31 & 248 & 5 & 261 & 28 \\
\hline & & & RERR & 12 & 247 & 5 & $146(240)$ & $122(32)$ \\
\hline & & & NI1 & 3 & 244 & 3 & 244 & 27 \\
\hline & & & NI2 & -110 & 292 & 24 & 271 & 39 \\
\hline \multirow[t]{8}{*}{10} & 0 & $L$ & BD & 7 & 276 & 0 & 263 & 21 \\
\hline & & & UW & 440 & 513 & 86 & 259 & 197 \\
\hline & & & WT & 26 & 298 & 8 & 286 & 32 \\
\hline & & & RE & 175 & 318 & 15 & 278 & 56 \\
\hline & & & RWRE & 26 & 302 & 10 & 307 & 22 \\
\hline & & & RERR & 8 & 283 & 3 & $198(272)$ & $98(32)$ \\
\hline & & & NI1 & 9 & 281 & 2 & 291 & 22 \\
\hline & & & NI2 & -857 & 992 & 260 & 490 & 186 \\
\hline \multirow[t]{8}{*}{10} & 0.5 & $H$ & BD & 10 & 247 & 0 & 249 & 26 \\
\hline & & & UW & 675 & 705 & 185 & 202 & 466 \\
\hline & & & WT & 371 & 420 & 70 & 201 & 232 \\
\hline & & & RE & 390 & 435 & 76 & 211 & 233 \\
\hline & & & RWRE & 371 & 420 & 70 & 214 & 209 \\
\hline & & & RERR & 357 & 407 & 64 & $143(195)$ & 332 (223) \\
\hline & & & NI1 & 351 & 403 & 63 & 225 & 170 \\
\hline & & & NI2 & 28 & 253 & 2 & 234 & 42 \\
\hline \multirow[t]{8}{*}{10} & 0.5 & $L$ & $\mathrm{BD}$ & 19 & 267 & 0 & 263 & 22 \\
\hline & & & UW & 680 & 719 & 170 & 230 & 417 \\
\hline & & & WT & 375 & 450 & 69 & 250 & 183 \\
\hline & & & $\mathrm{RE}$ & 506 & 553 & 108 & 241 & 270 \\
\hline & & & RWRE & 378 & 454 & 70 & 267 & 163 \\
\hline & & & RERR & 352 & 426 & 60 & $196(236)$ & 224 (177) \\
\hline & & & NI1 & 351 & 427 & 60 & 271 & 133 \\
\hline & & & NI2 & -406 & 589 & 121 & 466 & $\begin{array}{c}33 \\
\text { (continued) }\end{array}$ \\
\hline
\end{tabular}


Table 2 (continued)

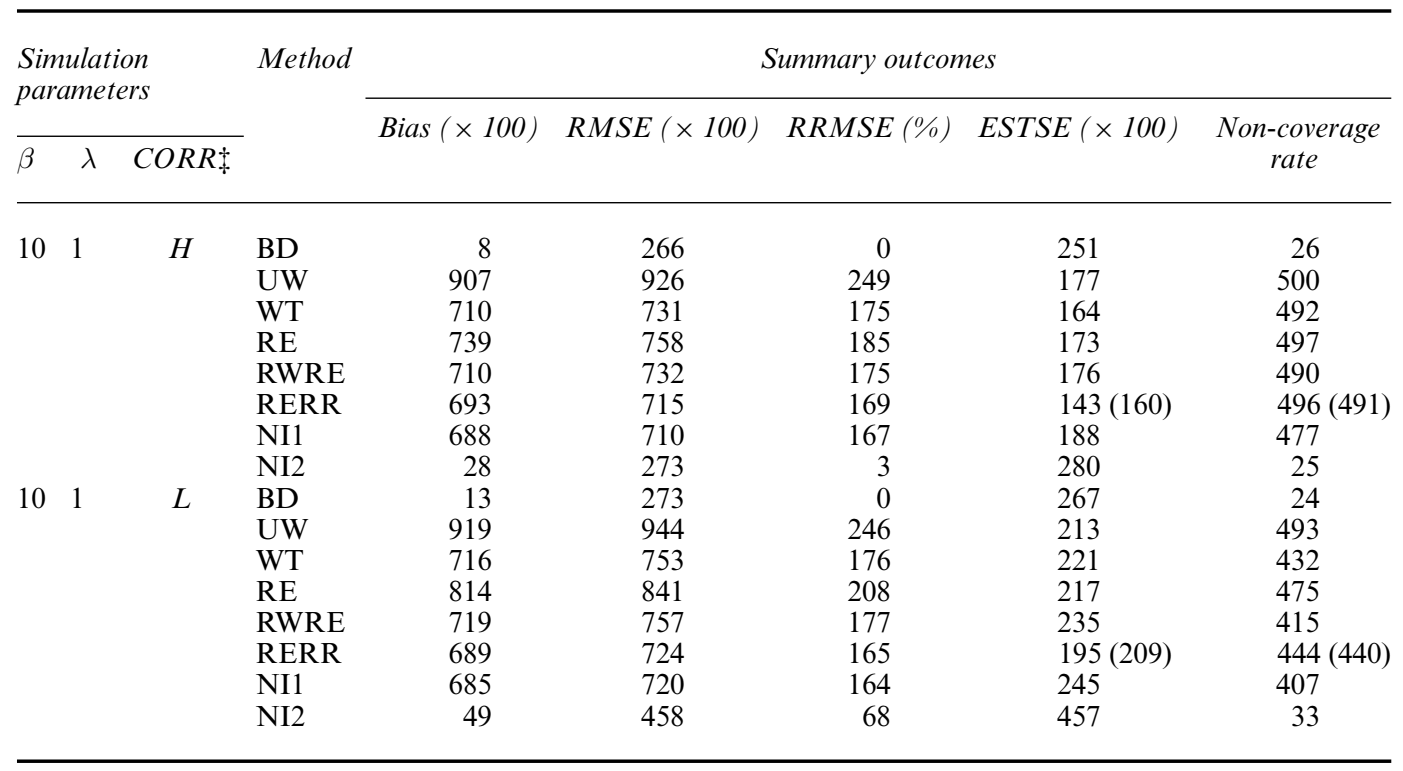

$\dagger$ Values in parentheses are the corresponding quantities from the bootstrap.

$\ddagger H$, high; $L$, low.

RRMSE $=100\{$ RMSE(estimator) - RMSE(BD) $\} /$ RMSE(BD),

the average estimated standard error ESTSE, which is the average of the estimated standard errors over the 500 samples, and $95 \%$ confidence interval non-coverage rate (nominally, we expect 25). Table 2 shows the results for the $(20,40)$ design.

\subsubsection{Missingness completely at random $(\beta=0)$}

All methods are consistent when the non-response mechanism is MCAR. RE has the smallest RMSE, reflecting gains in efficiency from shrinkage. UW, WT, RWRE, RERR and NI1 are comparable in terms of RMSE and coverage. NI2 has satisfactory coverage but is less efficient than the other methods.

\subsubsection{Cluster-specific non-ignorable non-response $(\beta=10, \lambda=0)$}

The missingness of $y_{i j}$ depends only on the cluster mean $\chi_{i}$ in this CSNI non-response case. UW has large bias and RMSE because of the lack of a non-response adjustment. WT has a slight bias and undercoverage for the $(80,10)$ design, which comes from dropping clusters with no respondents. RE has a substantial bias and poor coverage in this case since the missing data mechanism is not MCAR as discussed in Section 2.2. The bias of RE is larger when the within-cluster correlation is low and the number of SSUs is small. RWRE corrects or nearly corrects the bias of RE and has very similar performance to WT. It also has a slight bias and undercoverage for the $(80,10)$ design, since RWRE drops clusters with no respondents.

As expected, NI1 has the best performance in terms of RMSE and coverage in this situation, since it is correctly specified. RERR has a very similar performance to NI1 in terms of RMSE, suggesting that it is a useful approximate method when non-response depends on clusters. RERR not only corrects the bias of RE but also has smaller RMSE than WT. However, 
the model-based estimate of variance of RERR leads to confidence intervals that undercover the population mean. The bootstrap variance estimate leads to coverages that are closer to the nominal rate, suggesting that the bootstrap variance estimate is robust to the model misspecification that is caused by the approximation. NI2 has large bias and RMSE, especially when the within-cluster correlation is low, suggesting the sensitivity of this method to the misspecification of the missingness mechanism.

\subsubsection{Outcome-specific non-ignorable non-response $(\beta=10, \lambda=1)$}

In this OSNI non-response situation, non-response is related to individual outcomes within clusters, and NI2 is the only method that correctly specifies the OSNI mechanism. As a result, NI2 is the best method in term of RMSE and coverage, and the other methods all perform poorly, although RERR and NI1 outperform WT, RE and RWRE in terms of bias and RMSE.

\subsubsection{Mixed cluster-specific and outcome-specific non-ignorable non-response $(\beta=10, \lambda=0.5)$}

This mixed CSNI and OSNI non-ignorable missingness mechanism is somewhere between the above two extreme cases with $\lambda=0$ and $\lambda=1$. None of the methods are satisfactory, although NI2 does relatively well in the case of high within-cluster correlation. Thus there is no single method that dominates the others consistently over all simulation conditions.

\section{Application}

The Detroit Research Center on Oral Health Disparities at the University of Michigan School of Dentistry (also known as the Detroit Dental Health Project) is a multidisciplinary project that seeks to understand how the broader social context in which families live affects their oral health and well-being, and to design interventions that can have a positive effect within these contexts. The principal theme of the project is to investigate why some African-American children and their main care givers have better oral health than others who live in the same community.

A two-stage equal probability sample was drawn from the 392000 census tracts within Wayne County, Michigan, with the highest proportion of African-American households with income less than twice the poverty threshold. Blocks within each targeted tract were listed by tract and block number and a probability proportional to size selection method was implemented to select blocks for listing. A total of 118 blocks were selected across the 39 tracts. At the second stage, housing units within segments were selected by simple random sampling with the selection probabilities such that each housing unit has equal probability of selection, resulting in 12655 housing units selected for screening. The two main eligibility criteria were

(a) the household had at least one African-American child under the age of 6 years living in the household and

(b) the family's income is below $250 \%$ of the poverty level.

Of 12655 selected households, 1386 households were eligible and were invited to participate in the study. The families who agreed to participate in the study were then asked to schedule an appointment to come to the Dental Assessment Center, where they completed an interview questionnaire and clinical dental examination. During this process, unit non-response occurred either when households refused to participate in the study or when households who agreed to participate missed their appointment at the Dental Assessment Center. The unit non-response 


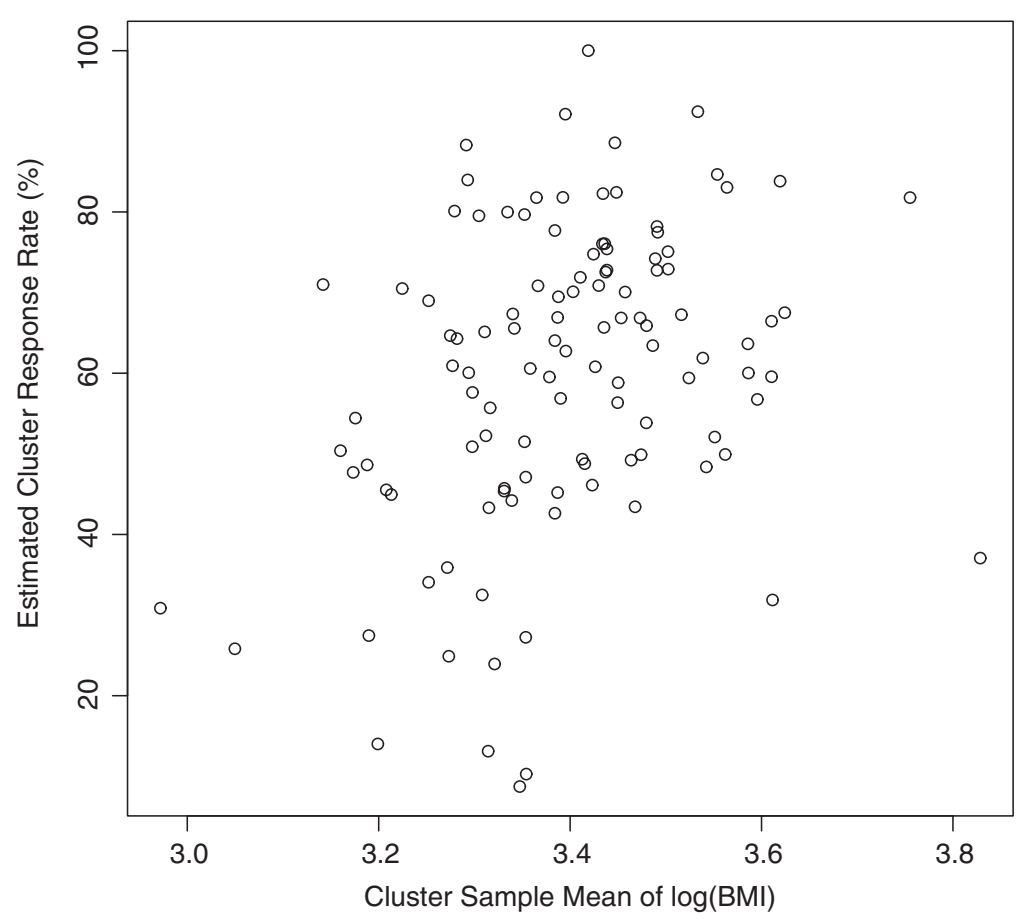

Fig. 1. Sample cluster mean of $\log (\mathrm{BMI})$ against estimated cluster response rate

rate was about 40\%. Item non-response arose when households failed to answer some questions in the questionnaire but, for most questions, the item non-response rate was less than $5 \%$.

We are interested in estimating the finite population mean of the body mass index BMI. The estimated cluster response rates for BMI range from $0 \%$ to $100 \%$, and the overall sample response rate is $56.2 \%$. To assess the relationship between cluster response rates and cluster means, we plot cluster sample response rates against cluster sample means of $\log (\mathrm{BMI})$. Fig. 1 displays a slightly linear trend with correlation coefficient 0.32 , suggesting that the non-response mechanism is not MCAR, and a CSNI or OSNI mechanism is indicated. This motivates us to apply our methods to estimate the finite population mean of BMI. The logarithm transformation is used to improve the normality of BMI data. Table 3 gives estimates of the finite population mean of $\log (\mathrm{BMI})$ and associated standard errors for various methods. All methods except NI2 yield similar results, and the UW and RE methods do not appear seriously biased in this example. RERR and WT appear somewhat less precise than other methods. The NI2 method has considerably higher standard error than the other methods. In this study, non-respondents are households who refused to participate in the study, or households who agreed to participate but missed their appointment at the Dental Assessment Center. We do not have any data for these non-respondents. From a statistical point of view, we cannot distinguish a CSNI or OSNI missing data mechanism on the basis of observed data. In this case, the comparison between NI1 and NI2 provides a form of sensitivity analysis for the CSNI and OSNI mechanisms of non-response. The estimates of mean BMI from NI1 and NI2 are 29.8 and 20.6 respectively. Instead of presenting a point estimate, we may report this range as the estimate. Unfortunately, for this particular study, this is unsatisfactory since, according to the criteria of the Centers for Disease Control and Prevention, a BMI of 20.6 belongs to the healthy category whereas a BMI of 29.8 belongs to the overweight category. 
Table 3. Estimates of the finite population mean of $\log (\mathrm{BMI})$ by various methods for the oral health disparities data $\dagger$

\begin{tabular}{|llllllll|}
\hline Estimate & \multicolumn{7}{|c|}{ Results for the following methods: } \\
\cline { 2 - 8 } & UW & $W T$ & RE & RWRE & RERR & NII & NI2 \\
\hline$\hat{\bar{Y}}$ & 3.404 & 3.392 & 3.403 & 3.394 & 3.390 & 3.394 & 3.024 \\
$\operatorname{ESTSE}(\hat{\bar{Y}})$ & 0.0079 & 0.0103 & 0.0076 & 0.0078 & $0.0125(0.0128)$ & 0.0100 & 0.0265 \\
\hline
\end{tabular}

$\dagger \hat{\bar{Y}}$ is the estimate of the population mean of $\log (\mathrm{BMI})$, and $\operatorname{ESTSE}(\hat{\bar{Y}})$ is the estimate of the associated standard error. The value in parentheses is the corresponding quantity from the bootstrap.

Although we cannot distinguish CSNI and OSNI statistically, we may understand the missing data mechanism from behavioural origins of non-response. In this particular study, the survey design requires fieldworkers to try to ask for the reason of refusal when eligible households refuse to participate in the study. Although only part of these households give the reason for refusal, we note that one major reason that eligible households refused to participate in the study is that they had jobs providing dental insurance. Since higher education is associated with lower BMI, and households with higher education are more likely to have a job with dental insurance, we might expect a positive relationship between BMI and response rate. Because education is an individual characteristic, we speculate that non-response is more likely to be related to an outcome variable, and non-response is OSNI non-response. If we have to choose a single model to report, we may favour the NI2 model.

Since this example did not give much indication of bias, we also ran a simulation under the CSNI mechanism that yielded a correlation between the outcome and the response rate which was comparable with that in this data set, and we compared the NI1 and UW estimates over 500 repeated samples. The UW method exhibited bias and undercoverage overall, but the estimates for the two methods were similar for a substantial fraction of the data sets as shown by Fig. 2, illustrating that properties cannot be inferred from the results of a single data set.

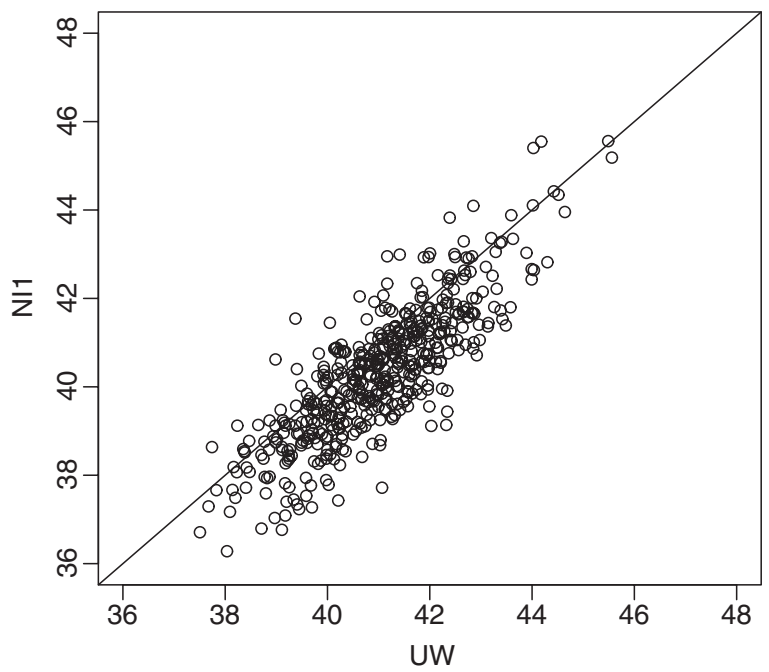

Fig. 2. UW estimates against NI1 estimates for 500 samples from the population with CSNI non-response 


\section{Conclusion}

This paper proposes several model-based estimates of the finite population mean for two-stage samples with unit non-response and compares them with existing methods by a simulation study. When the non-response mechanism is MCAR, all methods are consistent, but RE has the smallest RMSE owing to gains in efficiency from shrinkage. When non-response depends on cluster means, UW and RE are subject to bias. WT is consistent but can be inefficient if cluster response rates are variable. RWRE is a simple method to correct the bias of RE when non-response depends on clusters, but, like WT, it may fail in the presence of clusters without respondents. RWRE and WT have similar performances since the reweighting procedure in RWRE is to adjust weights of responses to reflect the correct weights, following the same reasoning as for WT. This similarity is especially pronounced in equal selection probability designs as in our simulation studies. In this case, the estimate of population mean in equation (6) is the same as WT since the adjusted $\kappa_{i}$ in equation (7) is constant across clusters. RERR is an approximate method to correct the bias of $\mathrm{RE}$ when non-response depends on clusters. It overcomes the drawback of RWRE and also gives a solution to the potentially low inefficiency of WT by borrowing strength across clusters in this case. In our simulations, RERR has a smaller RMSE than RE, RWRE and WT. A more rigorous extension of RE when nonresponse depends on clusters is NI1, which yields estimates that are similar to those from RERR. If the non-response depends on survey outcome, UW, WT, RE, RWRE, RERR and NI1 all have large bias and large RMSE. NI2 addresses this problem and yields consistent estimates. Although non-ignorable response models like NI1 and NI2 are attractive, we should be cautious in applying them because they are sensitive to the misspecification of the missing data mechanism, the normality assumption of the latent variable and the functional form between the outcome variable and the latent variable. Since these assumptions are untestable on the basis of the observed data, subject-matter knowledge or external information may be required to justify the assumptions.

A natural and important question given these findings is whether we can determine whether the non-response mechanism is MCAR, CSNI or OSNI, and which model should be used. A plot of cluster sample response rates against cluster sample means is useful for helping to understand the relationship between cluster response rates and cluster means. More formally, we can fit a linear regression model or nonparametric smooths and can test the relationship between cluster response rates and cluster means. If there is no significant relationship between cluster response rates and cluster means, RE may be justified. If the relationship is linear, then RERR may be used to reflect this relationship. If the relationship is non-linear, we may consider modelling it by using polynomial or spline techniques. Unfortunately, we cannot distinguish non-response depending on cluster means from non-response depending on outcomes from the plot. However, if auxiliary variables for non-respondents and respondents are available, e.g. from census data, then we could compare the distribution of auxiliary variables of non-respondents with that of respondents within a cluster. If there is no systematic difference, we might assume that non-response is more likely to depend on cluster means and apply WT, RWRE, RERR or NI1; otherwise, we may consider NI2. Since we do not have enough information to distinguish between alternative non-ignorable missingness mechanisms, it may be more appropriate to apply more than one method and to compare results.

Given the fact that alternative non-ignorable missing data mechanisms like CSNI and OSNI cannot be distinguished solely on the basis of observed data, the cognitive and social psychological theory of survey participation provides an important way to understand the nonignorable non-response mechanism (Groves and Couper, 1998; Krosnick, 1991). Non-ignorable 
non-response can arise if the cause of the non-response is the value of the survey variable itself or when causes of non-response are also causes of survey variables. The former case is most likely in self-administered questionnaire surveys. With this survey design, the householders have the opportunity to review the entire questionnaire before making the decision to participate. If the underlying cause of refusal to participate is the psychological threat that is posed by the topic in the survey, then the resulting non-response is most likely to be OSNI since the sample subjects' attributes on the survey variable are determining their probability of participating. In contrast, non-response in many interview-administrated surveys may be less directly related to the survey variables because the respondent has only a general idea about the topic of the survey. Nevertheless, as Groves and Couper (1998) noted, survey-specific characteristics may cause interview-administrated surveys to be specifically subject to OSNI non-response. For example, time use surveys measuring how people spend their time would be highly susceptible to non-contact non-response. Individuals who are away from home most of the time are more likely to be non-respondents through non-contact and are systematically different from others in 'away-from-home' activities, i.e. non-response directly depends on survey variables themselves.

It is probably more common that the survey variables that are measured do not cause nonresponse, but, instead, non-reponse and survey variables share a common possible unobserved cause. In these circumstances, CSNI non-response arises when the common cause is a cluster level characteristic (or covariate). If the common cause is an individual level characteristic (or covariate), OSNI non-response results. An example of this might be a two-stage cluster sample with schools as PSUs and students as SSUs. If non-response of students is deemed to be related to school level variables (e.g. school location, financial situation or goodness of administration) that are also associated with survey variables of interest, then the non-response is CSNI. However, if the non-response of students depends on individual characteristics such as a student's personality or academic performance, which also affect the survey variables, then the non-response is OSNI. Of course, if the common cause variable is known for both respondents and non-respondents, by conditioning on it, the non-ignorability of non-response can be largely removed. When designing and implementing surveys, it is important to anticipate systematic relationships between attributes of the survey request, the participant's decision to participate and the survey variable of interest, both for reduction of non-response and post-survey non-response adjustment (Groves and Couper, 1998). Understanding these relationships greatly helps us to identify CSNI and OSNI non-response and to choose appropriate models like NI1 or NI2 to achieve valid inference.

This paper focuses on two-stage cluster samples, but our models can be readily extended to accommodate multistage cluster samples. To reflect the design feature of multistage cluster sampling, we can use multilevel hierarchical models where different level within-cluster correlations are modelled by different level random effects. In this case, CNSI and OSNI non-response arise when non-response depends on clusters' characteristics or survey outcomes respectively. It is straightforward to adjust for OSNI non-response. The same latent variable approach as NI2 can be used to model the OSNI missing data mechanism. For CSNI non-response, the situation is a little complex. In the context of multilevel hierarchical models, CSNI non-response can associate with underlying cluster characteristics at different levels. In principle, we could extend the NI1 model to allow the mean of outcome variable to depend on random effects of all levels. However, since substantial within-cluster correlation may occur in only certain levels and also for the convenience of estimation, it may be adequate just to model the relationship between outcome variables and random effects of these levels.

In our simulation, the gains in efficiency of RE or RERR are modest. To focus on unit non-response adjustments in this paper, our simulations adopted an equal selection probability 
design and the weights are solely attributable to non-response. However, from equation (2), it is clear that if there are sampling weights, as in many survey designs, WT will be more variable; then the RE and RERR methods have greater potential for gains in efficiency. For simplicity, we have considered random-effects models that condition on cluster response rates alone, since this relationship is particularly important from the point of view of a consistent estimate of the population mean (Little and An, 2004). Other cluster level characteristics could be added to our models as covariates if available. In particular, we have assumed here that the size variable determining the first-stage selection probability is unrelated to the outcome. This assumption can be relaxed by including the size variable as a covariate in the random-effects model.

\section{Acknowledgements}

This paper was supported by grant DMS 0106914 from the National Science Foundation. The authors thank Amid I. Ismail at the School of Dentistry at the University of Michigan for providing the Detroit dental health data. The authors also thank three referees and the Associate Editor for their insightful and detailed comments that improved this paper.

\section{Appendix A: Gibbs sampler}

Let $\mathbf{y}_{\text {obs }}$ and $\mathbf{y}_{\text {mis }}$ denote values of the survey outcome $Y$ for respondents and non-respondents, and $\mathbf{z}=\left\{z_{i j}\right\}, i=1, \ldots, n, j=1, \ldots, m_{i}$, denote values of the latent variable. Consider the $k$ th iteration of the Gibbs sampler. For non-ignorable response model NI2, the first step of the iteration is 'data augmentation' (Tanner and Wong, 1987), in which the missing $y_{i j}$ and latent variable $z_{i j}$ are generated from their full conditional distributions. When $r_{i j}=1$, i.e. $y_{i j}$ is observed, $z_{i j}$ is drawn from the following left-truncated normal distribution $\mathrm{TN}$ :

$$
\left[z_{i j} \mid \mathbf{y}_{\mathrm{obs}}, r_{i j}=1, \alpha_{i}, \beta, \chi_{i}, \sigma^{2}\right]=\mathrm{TN}_{\left[z_{i j}>0\right]}\left\{\chi_{i}+\frac{\beta}{\beta^{2}+\sigma^{2}}\left(y_{i j}-\alpha_{i}-\beta \chi_{i}\right), \frac{\sigma^{2}}{\beta^{2}+\sigma^{2}}\right\} .
$$

When $r_{i j}=0,\left(y_{i j}, z_{i j}\right)$ are drawn from the conditional distributions

$$
\begin{aligned}
& {\left[z_{i j} \mid \mathbf{y}_{\text {obs }}, r_{i j}=0, \alpha_{i}, \beta, \chi_{i}, \sigma^{2}\right]=\operatorname{TN}_{\left[z_{i j}<0\right]}\left(\chi_{i}, 1\right),} \\
& {\left[y_{i j} \mid \mathbf{y}_{\text {obs }}, \mathbf{z}, r_{i j}=0, \alpha_{i}, \beta, \sigma^{2}\right]=N\left(\alpha_{i}+\beta z_{i j}, \sigma^{2}\right) .}
\end{aligned}
$$

Now, with augmented complete data $\left(\mathbf{y}_{\mathrm{obs}}, \mathbf{y}_{\mathrm{mis}}, \mathbf{z}\right)$, parameters are drawn alternately.

$\boldsymbol{\alpha}=\left\{\alpha_{i}\right\}, i=1, \ldots, n$, is drawn from the conditional distribution

$$
\left[\alpha_{i} \mid \mathbf{y}_{\mathrm{obs}}, \mathbf{y}_{\mathrm{mis}}, \mathbf{z}, \alpha, \beta, \sigma^{2}, \tau^{2}\right]=N\left\{\frac{m_{i} \tau^{2}\left(\bar{y}_{i}-\beta \bar{z}_{i}\right)+\sigma^{2} \alpha}{m_{i} \tau^{2}+\sigma^{2}}, \frac{\sigma^{2} \tau^{2}}{m_{i} \tau^{2}+\sigma^{2}}\right\}
$$

where

$$
\bar{y}_{i}=\frac{1}{m_{i}} \sum_{j=1}^{m_{i}} y_{i j}
$$

and

$$
\bar{z}_{i}=\frac{1}{m_{i}} \sum_{j=1}^{m_{i}} z_{i j}
$$

$\chi=\left\{\chi_{i}\right\}, i=1, \ldots, n$, is drawn from the conditional distribution

$$
\left[\chi_{i} \mid \mathbf{y}_{\mathrm{obs}}, \mathbf{y}_{\mathrm{mis}}, \mathbf{z}, \chi, \omega^{2}\right]=N\left(\frac{m_{i} \omega^{2} \bar{z}_{i}+\chi}{m_{i} \omega^{2}+1}, \frac{\omega^{2}}{m_{i} \omega^{2}+1}\right) .
$$

$\left(\beta, \sigma^{2}\right)$ are drawn from the conditional distributions 


$$
\begin{gathered}
{\left[\sigma^{2} \mid \mathbf{y}_{\mathrm{obs}}, \mathbf{y}_{\mathrm{mis}}, \mathbf{z}, \boldsymbol{\alpha}\right]=\operatorname{IG}\left\{a_{1}+\frac{1}{2}\left(\sum_{i=1}^{n} m_{i}-1\right), b_{1}+\frac{1}{2} \sum_{i=1}^{n} \sum_{j=1}^{m_{i}}\left(y_{i j}-\alpha_{i}-\hat{\beta} z_{i j}\right)^{2}\right\},} \\
{\left[\beta \mid \mathbf{y}_{\mathrm{obs}}, \mathbf{y}_{\mathrm{mis}}, \mathbf{z}, \boldsymbol{\alpha}, \sigma^{2}\right]=N\left\{\hat{\beta},\left(\sum_{i=1}^{n} \sum_{j=1}^{m_{i}} z_{i j}^{2}\right)^{-1} \sigma^{2}\right\}}
\end{gathered}
$$

where

$$
\hat{\beta}=\sum_{i=1}^{n} \sum_{j=1}^{m_{i}} z_{i j}\left(y_{i j}-\alpha_{i}\right) / \sum_{i=1}^{n} \sum_{j=1}^{m_{i}} z_{i j}^{2},
$$

and $\mathrm{IG}(\cdot)$ denotes an inverse gamma distribution.

$\left(\alpha, \tau^{2}\right)$ are drawn from the conditional distributions

$$
\begin{gathered}
{\left[\tau^{2} \mid \mathbf{y}_{\mathrm{obs}}, \mathbf{y}_{\mathrm{mis}}, \mathbf{z}, \boldsymbol{\alpha}\right]=\mathrm{IG}\left\{a_{2}+\frac{1}{2}(n-1), b_{2}+\frac{1}{2} \sum_{i=1}^{n}\left(\alpha_{i}-\bar{\alpha}\right)^{2}\right\},} \\
{\left[\alpha \mid \mathbf{y}_{\mathrm{obs}}, \mathbf{y}_{\mathrm{mis}}, \mathbf{z}, \boldsymbol{\alpha}, \tau^{2}\right]=N\left(\bar{\alpha}, \frac{\tau^{2}}{n}\right)}
\end{gathered}
$$

where

$$
\bar{\alpha}=\frac{1}{n} \sum_{i=1}^{n} \alpha_{i} .
$$

$\left(\chi, \omega^{2}\right)$ are drawn from the conditional distributions

$$
\begin{gathered}
{\left[\omega^{2} \mid \mathbf{y}_{\text {obs }}, \mathbf{y}_{\text {mis }}, \mathbf{z}, \chi\right]=\mathrm{IG}\left\{a_{3}+\frac{1}{2}(n-1), b_{3}+\frac{1}{2} \sum_{i=1}^{n}\left(\chi_{i}-\bar{\chi}\right)^{2}\right\},} \\
{\left[\chi \mid \mathbf{y}_{\text {obs }}, \mathbf{y}_{\text {mis }}, \mathbf{z}, \chi, \omega^{2}\right]=N\left(\bar{\chi}, \frac{\omega^{2}}{n}\right)}
\end{gathered}
$$

where

$$
\bar{\chi}=\frac{1}{n} \sum_{i=1}^{n} \chi_{i}
$$

\section{References}

Albert, J. and Chib, S. (1993) Bayesian analysis of binary and polychotomous response data. J. Am. Statist. Ass., 88, 669-679.

Amemiya, T. (1984) Tobit models: a survey. J. Econometr., 24, 3-61.

De Heer, W. (1999) International response trends: results of an international survey. J. Off. Statist., 15, $129-142$.

Gelfand, A. E., Hills, S. E., Racine-Poon, A. and Smith, A. F. M. (1990) Illustration of Bayesian inference in normal data models using Gibbs sampling. J. Am. Statist. Ass., 85, 972-985.

Gelman, A., Carlin, J. B., Stern, H. S. and Rubin, D. B. (2004) Bayesian Data Analysis, 2nd edn. New York: Chapman and Hall.

Gelman, A. and Rubin, D. B. (1992) Inference from iterative simulation using multiple sequences. Statist. Sci., 7, 457-511.

Groves, R. M. and Couper, M. P. (1998) Nonresponse in Household Interview Surveys. New York: Wiley.

Heckman, J. I. (1976) The common structure of statistical models of truncation, sample selection and limited dependent variables and a simple estimator for such models. Ann. Econ. Socl Measmnt, 5, 475-492.

Horvitz, D. G. and Thompson, D. J. (1952) A generalization of sampling without replacement from a finite population. J. Am. Statist. Ass., 47, 663-685.

Johnson, V. E. (1996) Studying convergence of Markov Chain Monte Carlo algorithms using coupled sampled paths. J. Am. Statist. Ass., 91, 154-166.

Kish, L. (1965) Survey Sampling. New York: Wiley.

Krosnick, J. A. (1991) Response strategies for coping with the cognitive demands of attitude measures in surveys. Appl. Cogn. Psychol., 5, 213-236.

Little, R. J. A. (2003) The Bayesian approach to sample survey inference. In Analysis of Survey Data (eds R. L. Chambers and C. J. Skinner), pp. 49-57. New York: Wiley. 
Little, R. J. A. (2004) To model or not to model?: competing modes of inference for finite population sampling. J. Am. Statist. Ass., 99, 546-556.

Little, R. J. A. and An, H. (2004) Robust likelihood-based analysis of multivariate data with missing values. Statist. Sin., 14, 949-968.

Little, R. J. A. and Rubin, D. B. (2002) Statistical Analysis with Missing Data, 2nd edn. New York: Wiley.

Little, R. J. A. and Vartivarian, S. (2002) On weighting the rates in nonresponse weights. Statist. Med., 22, $1589-1599$.

Rubin, D. B. (1976) Inference and missing data (with discussion). Biometrika, 63, 581-592.

Scott, A. and Smith, T. M. F. (1969) Estimation in multi-stage surveys. J. Am. Statist. Ass., 64, 830-840.

Sverchkov, M. and Pfeffermann, D. (2004) Prediction of finite population totals based on the sample distribution. Surv. Methodol., 30, 79-92.

Tanner, M. A. and Wong, W. H. (1987) The calculation of posterior distributions by data augmentation. J. Am. Statist. Ass., 82, 528-550. 